\title{
Clinical Characteristics of a Zoonotic Occupational Disease-Brucella endocarditis with Review of Literature
}

\author{
Vasanth Kataria Anilet ${ }^{1}$ Iragavarapu Tammiraju ${ }^{10}$ Meena Koduri ${ }^{1}$ \\ ${ }^{1}$ ASRAM Medical College, Eluru, Andhra Pradesh, India \\ Address for correspondence Tammiraju Iragavarapu, MD, DM, \\ Ind J Car Dis Wom 2021;6:235-240. \\ ASRAM Medical College, Eluru, Andhra Pradesh-534005, India \\ (e-mail: vmrtraju.mbbs@gmail.com).
}

\begin{abstract}
Keywords

- Brucella melitensis

- infective endocarditis

- mitral stenosis

- aortic valve abscess

- conservative

- valve replacement
\end{abstract}

Background Brucellosis is a zoonotic bacterial infection caused by a gram-negative aerobic coccobacillus. It can have varied presentation. Most severe complications include neurological and cardiovascular involvement (most commonly in the form of infective endocarditis). Aortic valve is most commonly involved (75\%). Blood culture is highly specific, while serologic tests are more sensitive for diagnosing brucellosis. Transthoracic and transesophageal echocardiography plays a major role in diagnosing infective endocarditis. Treatment of Brucella endocarditis includes combination of antibiotic therapy and surgical valve replacement. We are presenting here a case series of seven cases of $B$. endocarditis with different presentations and varied underlying cardiac conditions.

Materials and Methods Total seven cases of $B$. endocarditis were reported. The age group ranges from 22 to 45 years. Majority of them were from agriculture and dairy industry. All have presented with fever and three patients had severe breathlessness. Three cases were rheumatic valve diseases, one was bicuspid aortic valve, and one was congenital heart disease with prosthetic valve. Six patients had native valve endocarditis (four had predisposing cardiac condition with diseased valves, two had normal valves), whereas one had prosthetic valve endocarditis. Four had aortic valve involvement and three had mitral involvement. All cases were diagnosed using blood culture, serology, and echocardiography.

Conclusion All were treated initially with antibiotics and valve replacement was done in five cases. Mortality was the outcome in two cases on conservative treatment and before surgery. Other five patients recovered after surgery. published online January 25, 2022
DOI https://doi.org/

$10.1055 / \mathrm{s}-0041-1742213$. ISSN 2455-7854.
(C) 2022. Women in Cardiology and Related Sciences. All rights reserved.

This is an open access article published by Thieme under the terms of the Creative Commons Attribution-NonDerivative-NonCommercial-License, permitting copying and reproduction so long as the original work is given appropriate credit. Contents may not be used for commercial purposes, or adapted, remixed, transformed or built upon. (https://creativecommons.org/ licenses/by-nc-nd/4.0/)

Thieme Medical and Scientific Publishers Pvt. Ltd., A-12, 2nd Floor, Sector 2, Noida-201301 UP, India 


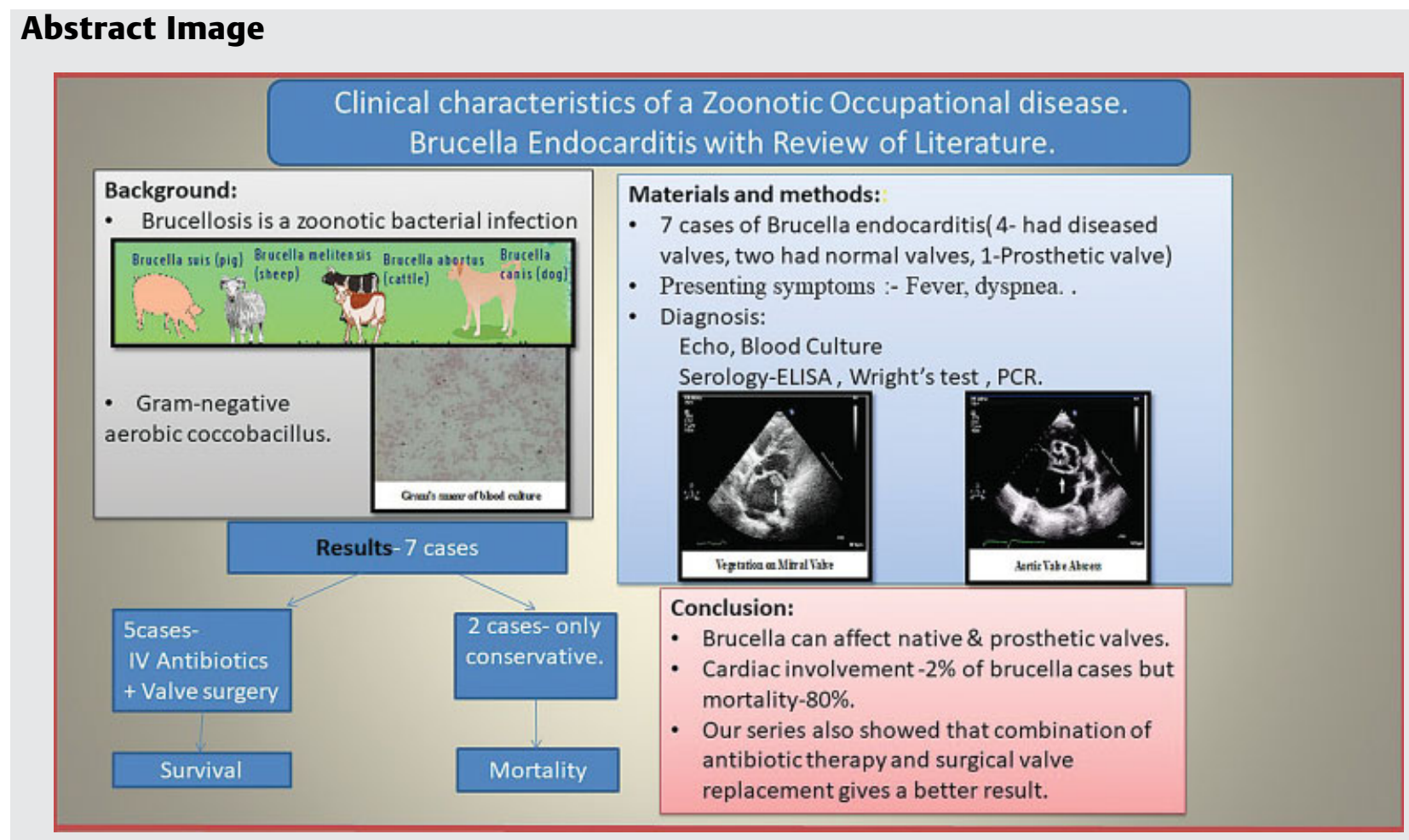

\section{Introduction}

Brucellosis is a zoonotic bacterial infection. Brucella melitensis, Brucella abortus, and Brucella suis are the three species of brucella mainly causing brucellosis. ${ }^{1}$ Brucella is a nonmotile gram-negative coccobacillus. It is intracellular and aerobic in nature. The disease is commonly seen in the Mediterranean region, Indian subcontinent, central and South America, and parts of the Soviet Union. It is mainly transmitted by consuming unpasteurized milk and cheese, inhalation of aerosolized infected particles, direct contact with infected animal parts, through skin cuts, or conjunctiva. ${ }^{2,3}$ Incubation period of brucella is $\sim 2$ to 6 weeks. ${ }^{4}$ Its incidence worldwide ranges from 0.03 to 160 per $100,000 .^{5}$

Presentation of brucellosis can be quite variable. It can range from mild-to-severe disease. Multiple organs and systems can be affected. Most severe and life-threatening complications include neurological and cardiovascular involvement (most commonly in the form of infective endocarditis). Most commonly involved is the aortic valve (75\%). ${ }^{4}$ In brucellosis, nearly $80 \%$ of the overall mortality is due to infective endocarditis. ${ }^{6-8}$ Mitral valve is less commonly affected. Prosthetic valve endocarditis too has been reported. $^{9}$

Table 1 The demographic characteristics, history of farm or animal exposure, predisposing factors, patient presentation, and the valve involved

\begin{tabular}{|l|l|l|l|l|l|l|}
\hline & Age $(\mathrm{y})$ & Sex & Farm exposure & Predisposing factors & Presentation & Valve involved, lesion \\
\hline Patient 1 & 44 & $\mathrm{M}$ & Business & CRHD & Fever & Severe MS \\
\hline Patient 2 & 45 & $\mathrm{M}$ & Agricultural labor & Bicuspid aortic valve & Fever & Severe AS, moderate AR \\
\hline Patient 3 & 32 & $\mathrm{M}$ & Farmer & CRHD & Fever, dyspnea & Severe MS, moderate MR \\
\hline Patient 4 & 36 & $\mathrm{M}$ & Not known & $\begin{array}{l}\text { No significant cardiac } \\
\text { pathology }\end{array}$ & Fever & Aortic valve abscess \\
\hline Patient 5 & 50 & $\mathrm{M}$ & Driver & CRHD & Fever, dyspnea & $\begin{array}{l}\text { Aortic valve perforation, } \\
\text { severe AR }\end{array}$ \\
\hline Patient 6 & 34 & $\mathrm{M}$ & Milk industry & Chronic kidney disease & Fever, dyspnea & Severe AR \\
\hline Patient 7 & 22 & $\mathrm{M}$ & Not known & $\begin{array}{l}\text { Acyanotic CHD, restrictive } \\
\text { ASD, VSD, congenital } \\
\text { severe MR }\end{array}$ & Fever, dyspnea & Prosthetic mitral valve \\
\hline
\end{tabular}

Abbreviations: AR, aortic regurgitation; AS, aortic stenosis; ASD, atrial septal defect; CHD, chronic heart disease; CRHD, chronic rheumatic heart disease; MR, mitral regurgitation; MS, mitral stenosis; VSD, ventricular septal defect. 


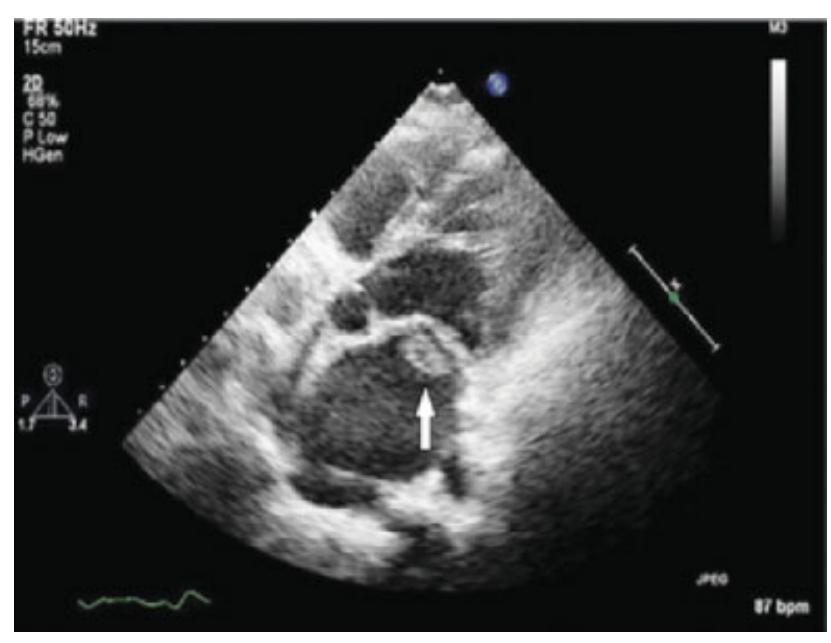

Fig. 1 Large vegetation on the mitral valve in patient with Brucella endocarditis (arrow).

We are presenting here a case series of seven cases of Brucella endocarditis with different presentations and varied underlying cardiac conditions. Also, we will be discussing about the diagnosis and management of B. endocarditis.

\section{Materials and Methods}

Our study includes total seven cases of $B$. endocarditis. The age group ranges from 22 to 45 years. All the seven patients are male. The demographic characteristics, history of exposure, predisposing factors, patient presentation, and the valve involved of all the above seven cases studied are summarized in -Table 1. Details of the patients are discussed in the following text:-

\section{Patient 1}

A 44-year-old male patient, known case of chronic rheumatic heart disease with severe mitral stenosis, presented with a history of fever for the last 2 months. Electrocardiogram (ECG) was suggestive of right bundle branch block. Echocardiogram showed severe mitral stenosis with large vegetation

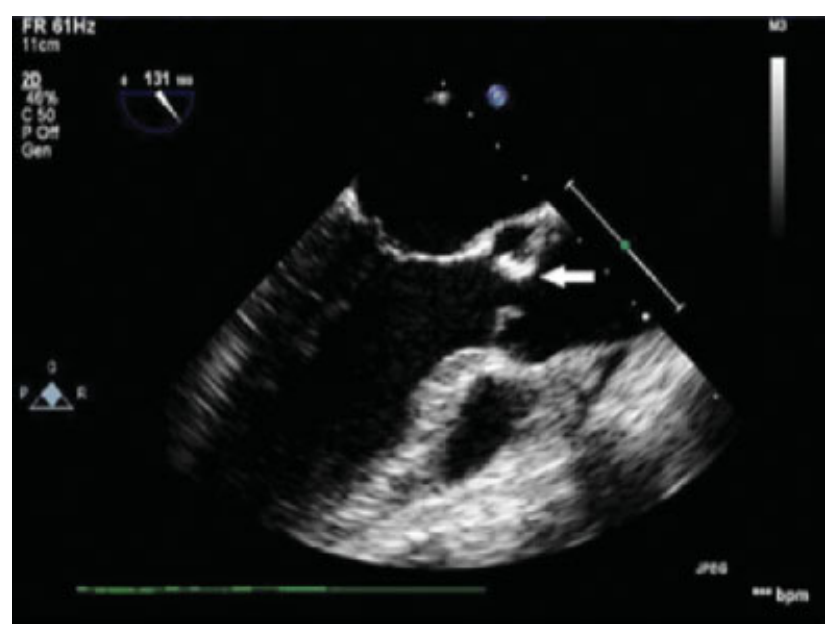

Fig. 2 Two-dimensional echo showing vegetation on bicuspid aortic valve (arrow).

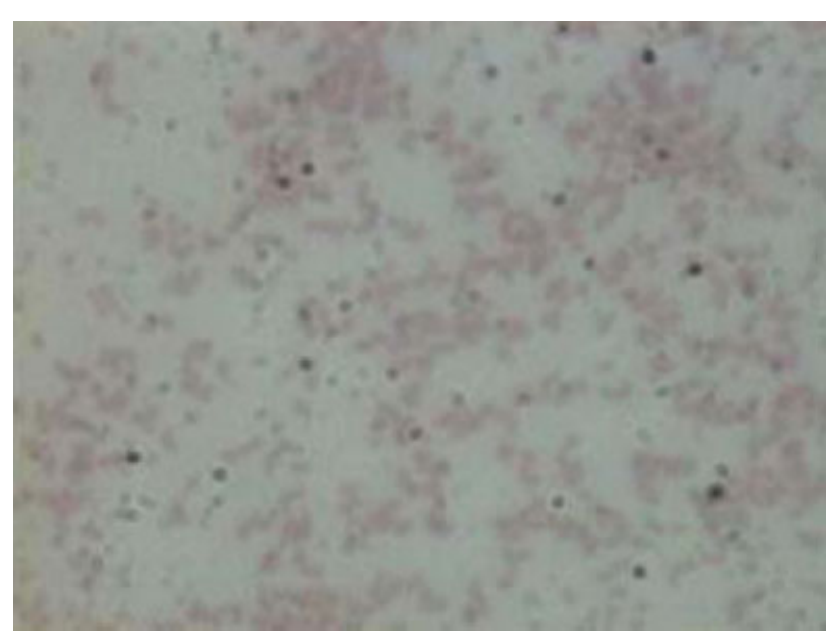

Fig. 3 Gram's smear of culture showing gram-negative Brucella melitensis bacillus.

on the mitral valve (-Fig. 1). His blood culture showed the presence B. melitensis. He was diagnosed to have B. endocarditis. He was treated initially with antibiotics. After stabilization, 3 months later he underwent elective mitral valve replacement.

\section{Patient 2}

The second patient was a 45-year-old male patient who was an agricultural labor by occupation. He was a known case of bicuspid aortic valve. He presented with chief complaints of fever for the last 2 months. ECG was suggestive of left ventricular hypertrophy. Echocardiography showed moderate sized vegetations on the aortic valve. He had severe aortic stenosis with moderate aortic regurgitation (-Fig. 2). Blood culture showed $B$. melitensis. Patient was treated successfully with antibiotics and valve replacement was done at a later date.

\section{Patient 3}

A 32-year-old male patient presented with complaints of continuous fever for the last 2 months and New York Heart Association (NYHA) class III breathlessness. He was a farmer by

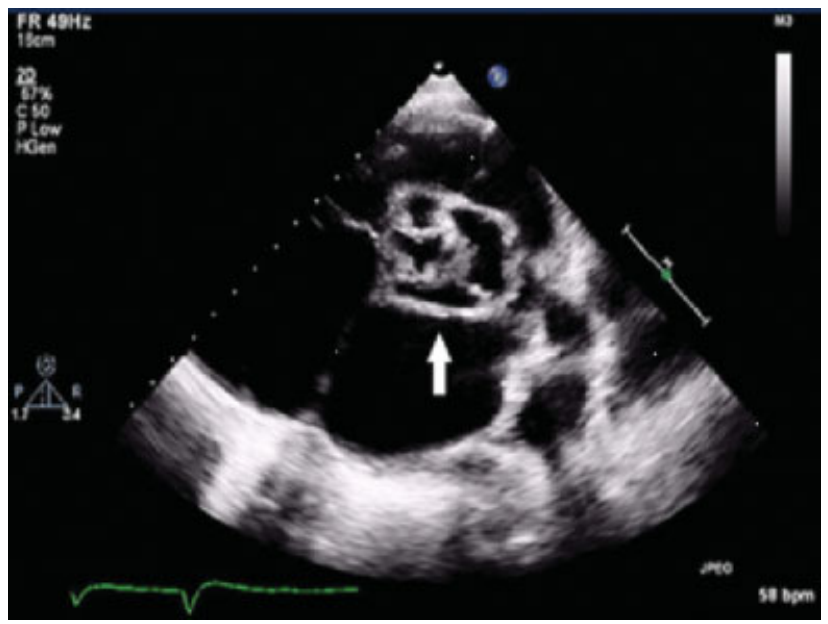

Fig. 4 Aortic valve abscess (arrow). 
occupation. Patient was a known case of chronic rheumatic heart disease with severe mitral stenosis, moderate mitral regurgitation, and severe pulmonary artery hypertension. He underwent percutaneous balloon mitral valvuloplasty 7 years back. At present, he had increased white blood cell count with raised C-reactive protein. ECG showed right bundle branch block. Echocardiogram was suggestive of moderate size vegetation on mitral valve leaflet. Positive blood culture confirmed the diagnosis of B. endocarditis (-Fig. 3). He was treated initially with medical management. After 3 months, he underwent mitral valve replacement.

\section{Patient 4}

A 36-year-old male patient came with chief complaints of pyrexia of unknown origin for the last 1 month. Routine blood investigations showed raised white blood cell counts. Echocardiography was suggestive of aortic valve abscess (-Fig. 4). Blood culture confirmed the presence of $B$. melitensis. Patient was treated with antibiotics and after 4 months he underwent aortic valve replacement.

\section{Patient 5}

A 50-year-old male presented with NYHA class II breathlessness with uncontrolled high-grade fever for 15 days. He was a known case of chronic rheumatic heart disease with mild mitral stenosis and mild aortic stenosis. ECG showed varying atrioventricular block. Echocardiography showed single $12 \mathrm{~mm}$ size vegetation on right coronary cusp of aortic valve with perforation in aortic valve, severe aortic regurgitation, and moderate pulmonary artery hypertension. Patient was diagnosed to have positive blood culture for Brucella. He was treated with antibiotics initially but patient did not respond to treatment and died while waiting for surgery.

\section{Patient 6}

A 34-year-old male, who works in milk industry, presented with fever for more than 3 weeks and NYHA class III breathlessness. He had prior history of chronic kidney disease. His ECG was suggestive of sinus tachycardia and echocardiography showed $9 \mathrm{~mm}$ size single vegetation on right coronary cusp of aortic valve with severe aortic regurgitation. Blood culture showed B. melitensis. He was treated with antibiotics and later he underwent aortic valve replacement. Patient is under regular follow-up.

\section{Patient 7}

The seventh patient was a 22-year-old male, a known case of acyanotic congenital heart disease. He had small atrial septal defect, moderate ventricular septal defect, and congenital severe mitral regurgitation. He underwent a patch closure and mitral valve replacement 3 years ago. He had a history of stuck prosthetic valve for which he was treated with thrombolytic therapy 1 year back. At present, he presented with severe breathlessness and continuous fever for the last 15 days. Echocardiogram showed significantly elevated gradients across the prosthetic valve. Blood culture showed B. endocarditis. He was treated with appropriate antibiotics. But patient did not respond to the treatment. He developed multiorgan dysfunction with shock and succumbed.

Empirical antibiotics given for all the cases include ceftriaxone $2 \mathrm{~g}$ and gentamicin $3 \mathrm{mg} / \mathrm{kg}$ prior to culture report. Agglutination tests were done that were positive with antibody titers elevated (1:640 - 1:1280). After confirmation of B. endocarditis in blood culture, treatment was started with doxycycline (100 mg twice daily), gentamicin ( $3 \mathrm{mg} / \mathrm{kg}$ intravenously in two divided doses), and rifampicin (600 mg once daily). Antibiotics were given for the period of 6 weeks.

\section{Results}

We have presented seven cases of $B$. endocarditis who were between 22 and 45 years of age. As all of them were male patients, sex differences in presentation, management, and disease outcome could not be studied. Three cases had history of exposure to animals. Five patients already had a predisposing cardiac condition like rheumatic heart disease, bicuspid aortic valve, and congenital heart disease. Four patients had aortic valve involvement, while three patients had mitral valve involvement. Six patients had native valve endocarditis, while one had prosthetic valve endocarditis. All were treated initially with antibiotics and valve replacement was done in five cases. Mortality was the outcome in two cases on conservative treatment and before surgery. Other

Table 2 Details of the treatment given and the patient outcome

\begin{tabular}{|l|l|l|l|l|}
\hline & Age & Sex & Treatment & Outcome \\
\hline Patient 1 & 44 & Male & IV antibiotics + elective MVR & Good \\
\hline Patient 2 & 45 & Male & IV antibiotics + AVR & Good \\
\hline Patient 3 & 32 & Male & IV antibiotics + elective MVR & Good \\
\hline Patient 4 & 36 & Male & IV antibiotics + AVR & Good \\
\hline Patient 5 & 50 & Male & IV antibiotics & Poor (Death) \\
\hline Patient 6 & Male & IV antibiotics + AVR & Good \\
\hline Patient 7 & 22 & Male & IV antibiotics & Poor (Death) \\
\hline
\end{tabular}

Abbreviations: AVR, aortic valve regurgitation; IV, intravenous; MVR, mitral valve regurgitation. 
five patients recovered after surgery and are under regular follow-up.

Treatment given and the patient outcome are summarized in -Table 2.

\section{Discussion}

Brucellosis is a zoonotic disease. Humans get this disease by direct contact with infected animals or their milk. Hence, farmers, workers, veterinarians, and laboratory personnel are at increased risk to get brucellosis. In our case series also three patients had exposure to infected animals or milk products. B. melitensis and B. abortus are predominantly responsible for causing endocarditis in $98 \%$ of cases. B. melitensis is more virulent and leads to more severe clinical course. ${ }^{9}$

Brucella initially produces a phase of generalized bacteremia. Then, it gets localized in the reproductive organs and reticuloendothelial system. Hence, it can affect multiple organs of the body and can have varied clinical manifestations. ${ }^{10}$ Clinical course in brucellosis can range from mild-tosevere disease. Brucellosis initially presents as febrile illness and can be associated with arthralgia, myalgia, and osteoarticular pain. Neurological complications including meningitis and encephalitis occur in 5 to $7 \%$ of cases. Most common complication of brucellosis leading to mortality is infective endocarditis. ${ }^{11,12}$ All the cases in our series presented with fever of prolonged duration. Dyspnea was present in those having severe valvular lesions.

Out of seven cases in our study, six patients had native valve endocarditis (four had predisposing cardiac condition with diseased valves, two had normal valves), whereas one had prosthetic valve endocarditis. Although infective endocarditis is less commonly seen with stenotic lesions, two of our cases had severe mitral stenosis with moderate mitral regurgitation as the predisposing cardiac condition.

In endemic areas, it is relatively easy to diagnose brucellosis; however, the areas where incidence of brucellosis is low, a high index of suspicion is needed. Various diagnostic methods available for brucellosis are culture, that is, whole blood or bone marrow culture, and serodiagnosis, that is, agglutination tests (Wright agglutination titer $>160$ ), enzyme-linked immunoassay (immunoglobulin A, immunoglobulin G, immunoglobulin M), 2-mercaptoethanol test and molecular detection (polymerase chain reaction). Blood culture is the only specific test. Brucella-positive blood culture with predisposing cardiac condition is usually equivalent to endocarditis. Serologic tests are more sensitive but less specific. They may be negative during the early stage of the disease. ${ }^{13}$ Brucella reproduces slowly and requires proper culture medium. Hence, even a negative blood culture does not rule out brucellosis. ${ }^{14}$ Transthoracic echocardiography plays a major role in detecting vegetations, ulcerations, and abscesses. ${ }^{15,16} \mathrm{~A}$ transesophageal approach may be required in few cases. In our case series also, blood culture, serology, and echocardiography were done for all the patients.

Although aortic valve is commonly involved, mitral valve involvement can be seen with previous structural changes such as rheumatic heart disease. Prosthetic valve endocarditis can cause complications like large vegetations, paravalvular leak, aneurysm, abscess, and valve malfunction. ${ }^{17}$ The most common complication is congestive heart failure (75-90\%). Even embolic events are frequently seen. ${ }^{18}$

Most effective therapy for $B$. endocarditis is a combination of antibiotic therapy and surgical valve replacement. Mortality rates in cases treated with antibiotic only is $32.7 \%$, while it is $6.7 \%$ in those treated with a combination of antibiotics and surgery. ${ }^{19}$ Antibiotic therapy must be started immediately after diagnosis. Shorter the time interval between disease onset and initiation of appropriate antibiotic therapy, better is the clinical outcome. The organism mainly causes tissue destruction with ulceration and increases the risk of embolization. Hence, the recommended antibiotic therapy consists of doxycycline, rifampicin, and aminoglycosides for 3 to 6 months. Surgical intervention, when indicated, should be performed after clinical stabilization. ${ }^{16}$ It is advisable to continue antibiotic therapy for 6 months after surgery. ${ }^{20}$

Indications for early surgery are presence of large vegetations, abscess, valve damage, and congestive heart failure. ${ }^{14}$ Even if there is symptomatic improvement with antibiotics, surgery can be still necessary to avoid embolic complication or for the relief of valvular obstruction. In our case series, five patients were managed on IV antibiotics initially that was followed by valve replacement. Two patients did not respond to treatment and succumbed.

\section{Conclusion}

All our seven cases of $B$. endocarditis had varied presentation and different underlying cardiac conditions. Brucella can affect both the native and prosthetic valves. Although cardiac involvement occurs in only $2 \%$ of brucella cases, it accounts for nearly $80 \%$ of the mortality. Diagnosis of B. endocarditis requires a high degree of suspicion. Blood culture, serological tests, and echocardiography play an important role in diagnosing $B$. endocarditis. Early diagnosis and appropriate management are essential for a successful outcome. Our series also showed that combination of antibiotic therapy and surgical valve replacement gives a better result.

\section{Audio 1}

Online content including video sequences viewable at: https://www.thieme-connect.com/products/ ejournals/html/10.1055/s-0041-1742213.

Note

No sources of research supporter funding, equipment, and drugs.

Conflicts of Interest

No conflicts of interest (of any) of the authors. 


\section{References}

1 Wise RI. Brucellosis in the United States. Past, present, and future. JAMA 1980;244(20):2318-2322

2 Hamieh A, Hamieh M. Brucella prosthetic valve endocarditis with septic and cardiogenic shock. IDCases 2020;21:e00881

3 Zheng R, Xie S, Lu X, et al. A systematic review and meta-analysis of epidemiology and clinical manifestations of human brucellosis in China. BioMed Res Int 2018;5712920:10

4 Reguera JM, Alarcón A, Miralles F, Pachón J, Juárez C, Colmenero JD. Brucella endocarditis: clinical, diagnostic, and therapeutic approach. Eur J Clin Microbiol Infect Dis 2003;22(11): 647-650

5 Bennett JE, Dolin R, Blaser MJ. Mandell, Douglas, and Bennett's Principles and Practice of Infectious Diseases. 8th edition Philadelphia, PA: Elsevier; 2015

6 Ferreira P, Gama P, Correia J, et al. Brucella endocarditis-case report and literature review. Rev Port Cardiol 2008;27(10): 1309-1315

7 Aygen B, Do ganay M, Su merkan B, Yildiz O, Kayabas U. Clinical manifestations, complications and treatment of brucellosis: a retrospective evaluation of 480 patients. Med Mal Infect 2002; 32:485-493

8 Fernández-Guerrero ML. Zoonotic endocarditis. Infect Dis Clin North Am 1993;7(01):135-152

9 Yavuz T, Ozaydin M, Ulusan V, Ocal A, Ibrisim E, Kutsal A. A case of mitral stenosis complicated with seronegative Brucella endocarditis. Jpn Heart J 2004;45(02):353-358

10 Young EJ. An overview of human brucellosis. Clin Infect Dis 1995; 21(02):283-289, quiz 290
11 Corbel M. Brucellosis in Humans and Animals: FAO, OIE, WHO. Geneva: WHO; 2006. Accessed December 28, 2021 from: http:// www.who.int/csr/resources/ publications/Brucellosis.pdf

12 Pappas G, Akritidis N, Bosilkovski M, Tsianos E. Brucellosis. N Engl J Med 2005;352(22):2325-2336

13 Köse S, Kiliç S, Ozbel Y. Identification of Brucella species isolated from proven brucellosis patients in Izmir, Turkey. J Basic Microbiol 2005;45(04):323-327

14 Berbari EF, Cockerill FR III, Steckelberg JM. Infective endocarditis due to unusual or fastidious microorganisms. Mayo Clin Proc 1997;72(06):532-542

15 Memish Z, Mah MW, Al Mahmoud S, Al Shaalan M, Khan MY. Brucella bacteraemia: clinical and laboratory observations in 160 patients. J Infect 2000;40(01):59-63

16 Delvecchio G, Fracassetti O, Lorenzi N. Brucella endocarditis. Int J Cardiol 1991;33(02):328-329

17 Keshtkar-Jahromi M, Boroumand M, Razavi SM, et al. Brucella endocarditis, a report of 14 cases (1991-2009). J Infect 2010;61(01):89-92

18 Mert A, Kocak F, Ozaras R, et al. The role of antibiotic treatment alone for the management of Brucella endocarditis in adults: a case report and literature review. Ann Thorac Cardiovasc Surg 2002;8(06):381-385

19 Keshtkarjahromi M, Razavi SM, Gholamin S, Keshtkarjahromi M, Hossain M, Sajadi M. Medical vs. medical and surgical treatment for brucella endocarditis: a review of the literature. Ann Thorac Surg 2012;94(06):2141

20 Tasdemir K, Kaya MG, Mavili E, et al. Surgical approach to the management of Brucella endocarditis. Eur J Cardiothorac Surg 2010;37(05):1021-1024 